\section{Detección de flavivirus en mosquitos (Diptera: Culicidae) de la Isla de Pascua-Chile}

\author{
Ximena Collao, Lorena Prado, Christian González , Ana Vásquez, \\ Romina Araki, Tuki Henríquez y Cindy Peña $M$.
}

\section{Detection of flavivirus in mosquitoes (Diptera: Culicidae) from Easter Island-Chile}

Flaviviruses are arthropod-borne viruses, mainly by mosquitoes of the genera Aedes and Culex (Culicidae) that are detected in tropical and subtropical areas. Main flaviviruses of public health importance are: dengue, West Nile virus, yellow fever, among others. In continental Chile, flaviviruses has not been detected. However, there are indigenous cases of dengue detected in Easter Island since 2002, as the presence of its vector Aedes aegypti. The aim of this study was: To determine diversity of flavivirus mosquitoes present in Easter Island. Thirty pools of mosquitoes collected in Hanga Roa were analyzed; a RT-PCR nested flavivirus was performed. Thirteen positive samples were detected and the amplification products were sequenced, identifying two specific flavivirus Insect, the Cell fusing agent virus and other related viruses Kamiti River. This is the first study in Chile showed the presence of flavivirus in vectors in Easter Island.

Key words: Flaviviruses, arboviruses, mosquitoes viruses.

Palabras clave: Flavivirus, arbovirus, virus de mosquitos.

\section{Introducción}

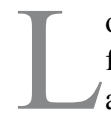

os flavivirus pertenecen a la familia Flaviviridae y, junto a otras familias y géneros, forman parte de los virus transmitidos por artrópodos conocidos como arbovirus ${ }^{1}$. Morfológicamente son virus icosahédricos, envueltos, de tamaño pequeño (30-40 nm). Poseen un ARN monocatenario de polaridad positiva de aproximadamente 11.000 nucleótidos que codifica una única poliproteína que da lugar a tres proteínas estructurales (C, M y E) y siete no estructurales (NS1, NS2A, NS2B, NS3, NS4A, NS4B, NS5) ${ }^{2}$.

El género Flavivirus comprende 53 especies²; entre las más conocidas, por causar enfermedades en el ser humano, se encuentran: dengue (DENV), fiebre amarilla (YFV) y el virus del Nilo Occidental (WNV). En general, estas enfermedades pueden presentarse como un cuadro febril inespecífico; infección del sistema nervioso central, incluidas encefalitis, meningitis

\footnotetext{
Universidad de Valparaíso. Escuela de Medicina, Laboratorio de Virología (XC, LP, CP). Centro Nacional de Microbiología, ISCIII, Madrid, España. Laboratorio de Arbovirus y Enfermedades Víricas Importadas (AV).

Instituto de Salud Pública de Chile. Sección Parasitología, Laboratorio de Entomología Médica (CG). Secretaría Regional Ministerial de Chile. Oficina Provincial de Isla de Pascua, Valparaíso (RA, TH).

Financiamiento: Laboratorio de Virología. Universidad de Valparaíso.

Los autores declaran no tener conflictos de interés.

Recibido: 7 de abril de 2014 / Aceptado: 23 de octubre de 2014

Correspondencia a:

Ximena Collao

ximena.collao@uv.cl
}

aséptica o mielitis o como una fiebre hemorrágica aguda, entre las manifestaciones más graves ${ }^{3}$.

La infección por DENV es una de las de mayor incidencia en la salud pública mundial y en la región de las Américas. Se han realizado grandes esfuerzos por controlar las epidemias que cada año se presentan. En el año 2013, se registraron más de 2 millones de casos de dengue en el mundo; 37.692 casos de dengue graves y 1.280 muertes por esta enfermedad ${ }^{4}$.

El virus se transmite por la picadura del mosquito Aedes aegypti. La enfermedad tiene presentaciones clínicas diferentes que van de estados benignos, hasta la evolución clínica grave, pudiendo causar la muerte. Existen cuatro serotipos: DENV-1, DENV-2, DENV-3 y DENV-4. La infección por un serotipo produce inmunidad permanente contra la reinfección por ese serotipo; sin embargo, la infección sucesiva con dos serotipos diferentes es un factor de riesgo para desarrollar las formas graves de la enfermedad ${ }^{4}$.

En el caso de la infección por YFV, ésta es endémica en África, América Central y Sudamérica. El YFV presenta tres ciclos de transmisión (selvático, intermedio y urbano), participando especies de mosquitos de los géneros Aedes y Haemagogus. En el ciclo urbano, el virus es transmitido por el mosquito Aedes aegypti. La enfermedad se caracteriza por falla hepática, renal, miocárdica y hemorragias generalizadas con una alta tasa de letalidad ${ }^{5}$. Tiene gran importancia en viajeros y para su prevención existe una vacuna.

El WNV fue conocido en el Nuevo Mundo en el año 1999 tras el brote en la ciudad de Nueva York $^{6}$, donde causó cuadros de encefalitis en la población. Desde esa fecha se ha distribuido ampliamente, y en el 2005, existían focos de transmisión sostenida en gran parte del hemisferio, con una distribución global que se extendió desde el centro de Canadá hasta el sur de Argentina ${ }^{7}$. El WNV es transmitido principalmente por la especie Culex pipiens y se mantiene en la naturaleza por la transmisión entre aves, pudiendo infectar a caballos y otros mamíferos. Las epidemias de mayor magnitud se han producido en E.U.A, Israel, Grecia, Rumania y Rusia. Los sitios donde se producen los brotes se encuentran a lo largo de las principales rutas de las aves migratorias ${ }^{8}$.

En el género Flavivirus existen también los denominados "insectoespecíficos", los cuales no se transmiten a vertebrados, debido a que replican sólo en células de mosquitos ${ }^{9}$.

Los principales vectores de los flavivirus son los culícidos (Diptera, Culicidae), comúnmente llamados mosquitos o zancudos. Se desarrollan en un amplio rango de comunidades bióticas, siendo, por lo general, más abundantes en zonas húmedas, tropicales y subtropicales. La clasificación de los culícidos reconoce dos subfamilias (Anophelinae y Culicinae), 44 géneros y más de 3.400 especies $^{10}$. En Chile se han citado 13 especies, cuatro géneros, y las dos subfamilias de culícidos. En la subfamilia Culicinae encontramos los géneros: Ochlerotatus Lynch-Arribálzaga, Aedes Meigen y Culex Linnaeus, el cual es el más diversificado ${ }^{11}$.

En Chile, en el año 2000 se detectó la presencia de $A$. aegypti y a principios del año 2002, se confirmó el primer caso autóctono de DENV-1. Ese año se detectaron 636 casos y no hubo fallecidos ${ }^{12}$. En los años 2012-2013 no se han detectado casos autóctonos en la Isla de Pascua ${ }^{13}$.

Tras detectar el vector se comenzó con la vigilancia entomológica en la zona y, posteriormente, con una vigilancia de casos febriles para diagnosticar la fiebre del DENV. Situación diferente es la de Chile continental, en que hubo reportes de infestación por $A$. aegypti ${ }^{14}$ hasta 1961. Actualmente se encuentra libre del vector.

En los últimos años se ha descrito que el aumento del turismo, el traslado de mercancías, la globalización, el cambio climático y la intensificación de los fenómenos meteorológicos extremos, como el de "El Niño" en 
Sudamérica ${ }^{15-17}$, producen cambios en el comportamiento de los vectores, con establecimiento de especies en lugares nunca antes registrados ${ }^{17-19}$. Es por esto que la amenaza de enfermedades infecciosas que puedan transmitir estos vectores en nuestro territorio se mantiene latente ${ }^{20-21}$.La presencia de casos autóctonos de DENV en la Isla de Pascua, y la circulación de su vector, se debe a múltiples factores, por lo que el estudio del tema y la vigilancia continua, tanto de los casos febriles como del vector, es de gran importancia. El objetivo principal de este estudio fue conocer la diversidad de los flavivirus presentes en mosquitos de la Isla de Pascua.

\section{Material y Métodos}

Recolección de muestras. En marzo de 2011 se recolectaron 30 lotes de larvas (10-20 larvas/lote) de mosquitos del poblado de Hanga Roa, para ello se utilizó el método de "muestreo de levantamiento de índices rápidos" (LIRA). Las muestras se recolectaron directamente desde las canaletas y recipientes artificiales encontrados en los patios de las casas y fueron preservadas en etanol al $80 \%$. Posteriormente, se enviaron a dos laboratorios del continente. Identificación taxonómica. Se basó en la utilización de la clave experimental descrita por Darsie Jr. R F y cols. ${ }^{22}$, y fue realizada en el laboratorio local de la Isla de Pascua. Su confirmación se realizó en el Laboratorio de Entomología Médica del Instituto de Salud Pública de Chile. El método consistió en un aclaramiento de las larvas con $\mathrm{NaOH}$ al $20 \%$ y posteriormente secadas en un baño termorregulador a $70^{\circ} \mathrm{C}$ por 20 minutos. Finalmente se realizaron dos lavados con alcohol al 70\%; se observaron en un microscopio Nikon Eclipse 80i y fueron registradas con el software ACT-2U. Detección de flavivirus. En el Laboratorio de Virología se almacenaron las muestras a $-80^{\circ} \mathrm{C}$ hasta su procesamiento. Previo a la obtención del material genético se maceraron las muestras en un tampón de lisis (Nuclisens, Biomeriéux), y luego se agitaron en un vórtex por 30 segundos. Seguido a esto, las muestras fueron centrifugadas (centrífuga Eppendorf) por 30 minutos a 15000 RPM. Se recolectó el sobrenadante y se utilizaron $300 \mathrm{uL}$ para la extracción del ARN viral. Se utilizó el método basado en la extracción con tiocianato de guanidina y sílica gel (Nuclisens Magnetic Extraction Reagents, Biomeriéux), utilizando el equipo Mininag (Biomeriéux). RPC anidada de flavivirus. Se utilizaron los partidores y condiciones descritas por Sánchez-Seco y cols. ${ }^{23}$. En la primera reacción se utilizó el AccessQuick ${ }^{\text {TM }}$ RT-PCR System. Se utilizó un termociclador PCR Sprint Thermal Cycler (Thermo Electron Corporation). Los productos de amplificación fueron analizados mediante electroforesis en gel de agarosa al $2 \%$ teñido con $2,5 \mu \mathrm{g} / \mathrm{mL}$ Red Gel (Genexpress) en buffer TBE (Trisborato-EDTA; $\mathrm{pH} 8,3$ ) (Promega) y examinados en un transiluminador (UVP DigiDoc-It ${ }^{\mathrm{TM}}$, BioImaging Systems). En cada reacción se utilizó un control positivo de ARN inactivado de DENV 1-4, gentileza del Dr. Antonio Tenorio (ISCIII, España), y como control negativo se utilizó agua libre de ARNasas y ADNasas. Secuenciación. Los productos de amplificación fueron purificados desde el gel utilizando el kit WIZARD SV Gel (Promega), y se enviaron a secuenciar ambas hebras del ADN (Macrogen, Korea). Identificación de flavivirus. Las secuencias obtenidas fueron analizadas con los programas Bioedit versión 7.2.5, Chromas versión 2.4 $4^{24,25}$ y posteriormente, se compararon con 30 secuencias, en su mayoría disponibles en el banco mundial de genes (NCBI), y algunas otras obtenidas por la gentileza de la Dra. Vásquez (ISCIII, España). Se utilizó el programa BLAST (Basic local Alignment Search Tool) y finalmente se realizó un árbol filogenético con el programa MEGA versión $5^{26}$.

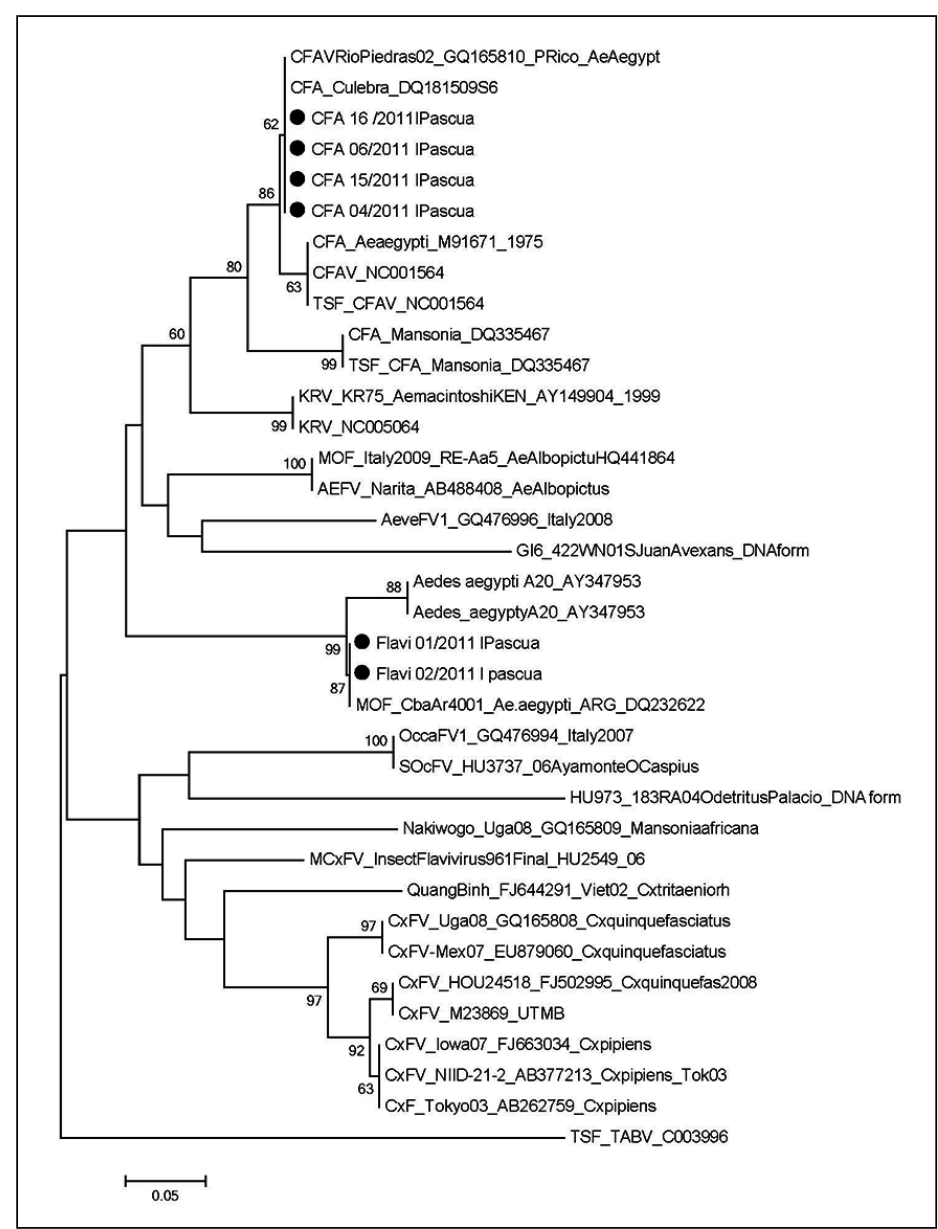

Figura 1. Árbol filogenético. Agrupamiento de las secuencias de flavivirus detectados en mosquitos de Isla de Pascua. Se analizaron 36 secuencias de flavivirus específicos de insectos con el método Neihgbor Joinig, distancia P, y 1.000 réplicas de Bootstrap. Las secuencias obtenidas en este estudio se observan con un círculo de color negro.

\section{Resultados}

En el presente estudio se detectaron 13 muestras positivas para flavivirus en mosquitos del género Aedes y Culex. Se obtuvieron seis secuencias entre 50-84 nucleótidos de la secuencia de la proteína no estructural NS5 de los flavivirus (Tabla 1). Tras su análisis se identificaron dos grupos de flavivirus específicos de insectos: cuatro secuencias se agrupaban con el virus cell fusing agent (CFAV), demostrando 99\% de similitud con cepas de CFAV detectadas en mosquitos de Puerto Rico, según el análisis tipo BLAST. Las otras dos secuencias detectadas se agruparon con secuencias detectadas en Ae. aegypti en Argentina y relacionada con Kamiti River Virus (Figura 1).

\section{Discusión}

Entre los flavivirus que afectan a hospedadores vertebrados, existen 27 transmitidos por mosquitos y 12 transmitidos por garrapatas, el resto no posee un vector artrópodo conocido. Dentro de este último grupo se encuen- 
Tabla 1. Secuencias de los flavivirus detectados en vectores de la Isla de Pascua

\begin{tabular}{|c|c|}
\hline Nombre & Secuencia $5^{\prime}-3^{\prime}$ \\
\hline $\begin{array}{l}\text { CFA 04/2011 } \\
\text { IPascua }\end{array}$ & $\begin{array}{l}\text { CCCAGCCTAGCTGGTGAGGCCAAGGGCTCAAGGACCATCTGGTAC } \\
\text { ATGTGGCTTGGGAGCCGGTTCCTTGAATTTGAGGCACTA }\end{array}$ \\
\hline $\begin{array}{l}\text { CFA 15/2011 } \\
\text { IPascua }\end{array}$ & $\begin{array}{l}\text { CCCAGCCTAGCTGGTGAGGCCAAGGGCTCAAGGACCATCTGGTAC } \\
\text { ATGTGGCTTGGGAGCCGGTTCCTTGAATTTGAGGCACTA }\end{array}$ \\
\hline $\begin{array}{l}\text { CFA 06/2011 } \\
\text { IPascua }\end{array}$ & $\begin{array}{l}\text { CCCAGCCTAGCTGGTGAGGCCAAGGGCTCAAGGACCATCTGGTAC } \\
\text { ATGTGGCTTGGGAGCCGGTTCCTTGAATTTGAGGCACTA }\end{array}$ \\
\hline $\begin{array}{l}\text { CFA 16/2011 } \\
\text { IPascua }\end{array}$ & $\begin{array}{l}\text { CCCAGCCTAGCTGGTGAGGCCAAGGGCTCAAGGACCATCTGGTAC } \\
\text { ATGTGGCTTGGGAGCCGGTTCCTTGAATTTGAGGCACTA }\end{array}$ \\
\hline $\begin{array}{l}\text { Flavi 01/2011 } \\
\text { IPascua }\end{array}$ & $\begin{array}{l}\text { CCGACCATAGCTGGGGAGCCGAAAGGATCCCGGACCATTTGGTAT } \\
\text { ATGTGGCTGGAAGTCGA }\end{array}$ \\
\hline $\begin{array}{l}\text { Flavi 02/2011 } \\
\text { IPascua }\end{array}$ & $\begin{array}{l}\text { CCGACCATAGCTGGGGAGCCGAAAGGATCCCGGACCATTTGGTAT } \\
\text { ATGTG }\end{array}$ \\
\hline
\end{tabular}

tran los flavivirus “insecto-específicos”, cuya descripción ha aumentado en los últimos años ${ }^{27-29}$, los cuales no tienen capacidad de replicar en células de vertebrados ${ }^{9}$. El primer virus descrito fue el CFAV, aislado del vector $A$. aegypti ${ }^{30}$. Varios estudios indican que este virus tiene un ancestro común con los flavivirus que pueden afectar al hombre ${ }^{31}$.

En relación a los dos grupos de secuencias detectadas en este trabajo, sería necesario amplificar una región distinta a la utilizada o bien detectar un fragmento de mayor tamaño, para concluir de manera más acabada acerca de la caracterización molecular y la filogenia de los datos obtenidos.

$\mathrm{Si}$ bien, no podemos concluir que se estudió ampliamente la diversidad de flavivirus en los vectores de la Isla de Pascua, debido al bajo número de muestras analizadas y al tiempo acotado de recolección, la detección de otros flavivirus no DENV da cuenta de que existe una variedad de flavivirus en vectores de Isla de Pascua, y que se requiere continuar con estos estudios, analizando un mayor número de muestras.

En las muestras analizadas de mosquitos se identificaron Ae.aegypti y Cx. pipiens. Sin embargo, debido a que existían mezclas en los lotes de muestras analizados, no fue posible identificar los vectores con resultados positivos, necesitándose de otros estudios entomológicos futuros que nos permitan concluir al respecto. Aun así, consideramos importante la detección de Cx. pipiens en la Isla de Pascua ya que es un conocido vector del WNV, está íntimamente relacionado con asentamientos humanos y es capaz de adecuarse muy bien a condiciones climáticas y espaciales en los distintos hábitat donde se desarrolla.

Este es el primer estudio de flavivirus realizado en vectores en Chile $\mathrm{y}$, si bien es preliminar, nos permite conocer la existencia de flavivirus autóctonos de los mosquitos de la Isla de Pascua y sus vectores principales, lo cual motiva a realizar futuras exploraciones en el tema, mejorando las limitaciones encontradas.

\section{Resumen}

Los flavivirus son virus transmitidos por artrópodos, principalmente por especies de mosquitos de los géneros Aedes y Culex (Culicidae) que se detectan principalmente en zonas tropicales y subtropicales. Los principales flavivirus de importancia en salud pública son el virus del dengue, del Nilo Occidental y fiebre amarilla, entre otros. En Chile continental no hay registro de flavivirus; sin embargo, se han detectado casos autóctonos de dengue en la Isla de Pascua desde el año 2002, al igual que la presencia de su vector Aedes aegypti. El objetivo de este estudio fue conocer la diversidad de los flavivirus presentes en mosquitos de la Isla de Pascua. Se analizaron 30 lotes de larvas de mosquitos de Hanga Roa, se realizó una RT-RPC anidada específica y genérica para flavivirus. Se detectaron 13 muestras positivas, identificándose dos flavivirus específicos de insectos, el virus cell fusing agent y otro relacionado con Kamiti river virus. Este es el primer estudio en Chile que evidencia la presencia de flavivirus en los vectores de la Isla de Pascua.

\section{Referencias bibliográficas}

1.- Karabatsos N. International catalogue of Arboviruses including certain other viruses of vertebrates. Am Soc Trop Med and Hyg. 1985. San Antonio, Texas: 3rd ed, pág 84-6.

2.- Chambers T J, Hahn C S, Galler R, Rice C M. Flavivirus genome organization, expression, and replication. Annu Rev Microbiol 1990; 44: 649-88.

3.- Calisher C H, Gould E A. Taxonomy of the virus family Flaviviridae. Adv Virus Res 2003; 59: 1-19.

4.- Organización Mundial de la Salud, Organización Panamericana de la Salud. Dengue. http://www.paho.org/hq/index.php?option=com_content\&view=arti cle\&id=264\&Itemid=363 (accedido: 17 de marzo de 2014).

5.- Monath T P. Yellow Fever: an update. Lancet Infect Dis 2001; 1: 11-20.

6.- Lanciotti R S, Roehrig J T, Deubel V, Smith J, Parker M, Steele K, et al. Origin of the West Nile virus responsible for an outbreak of encephalitis in the northeastern United States. Science 1999; 286: 2333-7.

7.- Gubler D J. The continuing spread of West Nile virus in the western hemisphere. Clin Infect Dis 2007; 45: 1039-46.

8.- Centers for Disease Control and Prevention. West Nile Virus in the United States: Guidelines for Surveillance, Prevention and Control. $4^{\text {th }}$ Revision, June 14, 2013: 7-10. http://www.cdc.gov/westnile/resources/pdfs/ wnvguidelines.pdf

9.- Kuno, G. Host range specificity of flaviviruses: correlation with in vitro replication. J Med Entomol 2007; 44: 93-101.

10.- Harbach R E. The Culicidae (Diptera): a review of taxonomy, classification and phylogeny. Zootaxa 2007; 1668: 591-638.

11.- González C R, Jercic M I, Muñoz L. Los culícidos de Chile (Diptera: Culicidae). Acta Entomológica Chilena 2005; 29: 31-5.

12.- Perret C, Abarca K, Ovalle J, Ferrer P, Godoy P, Olea A, et al. Dengue-1 virus isolation during first dengue fever outbreak on Easter Island, Chile. Emerg Infect Dis 2003; 11: 1465-7.

13.- Informe de Dengue (CIE 10: A90-A91) Semana Epidemiológica 1 a 52 (30/12/2012 al 28 de diciembre de 2013) http://epi.minsal.cl/epi/html/bolets/ reportes/Dengue/Dengue_SE522013.pdf

14.- Trincado A, Meléndez F, Mejías G, Bachler G, González J, Segura J, et al. Manual de Procedimiento Para la Vigilancia de Aedes aegypti. Programa de Prevención de Mosquitos y Vectores, Seremi de Salud, Región de Valparaíso, 2002. Chile.

15.- Heft D E, Walton W E. Effects of the El Niño-Southern Oscillation (ENSO) cycle on mosquito populations in southern California. J Vector Ecol 2008; 33:17-29.

16.- Linthicum K J, Anyamba A, Tucker C J, Kelley P W, Myers M F, Peters C J. Climate and satellite indicators to forecast Rift Valley fever epidemics in Kenya. Science 1999; 285: 397-400.

17.- López-Vélez R, Molina Moreno R. Cambio climático en España y riesgo 
de enfermedades infecciosas y parasitarias transmitidas por artrópodos y roedores. Rev Esp Salud Publica 2005; 79: 177-90.

18.- Avilés G, Rangeon G, Vorndam V, Briones A, Baroni P, Enria D, et al. Dengue reemergence in Argentina. Emerg Inf Dis 1999; 5: 575-8.

19.- Gratz N G. Critical review of the vector status of Aedes albopictus. Med Vet Entomol 2004; 18: 215-27.

20.- Lounibos L P. Invasions by insect vectors of human diseases. Annu Rev Entomol 2002; 47: 233-66.

21.- Sutherst R W. Global change and human vulnerability to vector-borne diseases. Clin Microbiol Rev 2004; 17: 136-73.

22.- Darsie Jr. R F. Mosquitoes of Argentina. Part I. Keys for identification of adult females and fourth stage larvae in English and Spanish (Diptera: Culicidae). Mosq Syst 1985; 17 (3): 153-283.

23.- Sánchez-Seco M P, Rosario D, Domingo C, Hernández L, Valdés K, Guzmán $\mathrm{M} \mathrm{G}$, et al. Generic RT-nested-PCR for detection of flaviviruses using degenerated primers and internal control followed by sequencing for specific identification. J Virol Methods 2005; 126: 101-9.

24.- Hall T A. BioEdit: a user-friendly biological sequence alignment editor and analysis program for Windows 95/98/NT. Nucleic Acids Symp Ser 1999; 41: 95-8.

25.- Technelsium PTy Ltd, Copyrigh 1998-2012. http://www.technelysium.com. au Fecha acceso:

26.- Tamura K, Peterson D, Peterson N, Stecher G, Nei M, Kumar S. MEGA5: Molecular evolutionary genetics analysis using maximum likelihood, evolutionary distance, and maximum parsimony method. Mol Biol Evol 2011; 28: 2731-9.

27.- Cook S, Moureau G, Harbach R E, Mukwaya L, Goodger K, Ssenfuka F, et al. Isolation of a novel species of Flavivirus and a new strain of Culex flavivirus (Flaviviridae) from a natural mosquito population in Uganda. $\mathrm{J}$ Gen Virol 2009; 90: 2669-78.

28.- Farfan-Ale J A, Loroño-Pino M A, García-Rejon J E, Soto V, Lin M, Staley $\mathrm{M}$, et al. Detection of flaviviruses and orthobunyaviruses in mosquitoes in the Yucatan Peninsula of Mexico in 2008. Vector Borne Zoonotic Dis 2010; 10: $777-83$.

29.- Morales-Betoulle M E, Monzón Pineda M L, Sosa S M, Panella N, López M R, Cordón-Rosales C, et al. Culex flavivirus isolates from mosquitoes in Guatemala. J Med Entomol 2008; 45: 1187-90.

30.- Stollar V, Thomas V L. An agent in the Aedes aegypti cell line (Peleg) which causes fusion of Aedes albopictus cells. Virology 1975; 64: 367-77.

31.- Gould E A, de Lamballerie X, Zanotto P M, Holmes E C. Origins, evolution, and vector/host coadaptations within the genus Flavivirus. Adv Virus Res 2003; 59: 277-314. 